\title{
Colonic lymphoid-glandular complex (microbursa): nature and morphology
}

\author{
W. F. KEALY
}

From the Departments of Morbid Anatomy, King's College Hospital, London and Kingston Hospital, Kingston-on-Thames, Surrey

SYNOPSIS A study of lymphoid-glandular complexes of the large bowel has been undertaken. Sections from 1924 surgical colectomy and proctocolectomy specimens were examined, and lymphoid-glandular complexes were observed in 231 . It has been shown that they are distributed throughout the large bowel and occur in all age groups and in normal and disease states. An analogy has been drawn between them, the palatine tonsils, and the bursa of Fabricius. It is concluded that the lymphoid-glandular complex is, most probably, a normal structural entity of the large bowel and that it acts as a local receptor of antigenic material for future immune recognition. It is suggested that microbursa rather than lymphoid-glandular complex is a more apt name for this structure.

The lymphoid tissue of the colon is distributed as lymphoid nodules or aggregates in close apposition to the muscularis mucosae, either below it in the upper part of the submucosa, above it in the lamina propria, lying between its muscle fibres or filling gaps in its substance. The overlying mucosa may be seen, at times, to protrude through these gaps in the muscularis mucosae to a variable extent in close association with the underlying lymphoid nodule with which it forms an intimate lymphoid-glandular (L-G) complex (fig 1). Clark $(1969,1970)$ described similar structures, referring to them as microdiverticula, and he discussed their importance in relation to granulomatous ileocolitis and ulcerative colitis. Dyson (1975) referred to them as glandular herniations and described a possible mode of their development in ulcerative colitis.

A study of L-G complexes of the large bowel has been undertaken in an effort to determine the nature of these structures.

\section{Methods}

Standard blocks for section, about $4 \mathrm{~cm}$ in length, were taken from the ascending, transverse, descending, and sigmoid regions of necropsy specimens of colon but autolysis hindered detailed examination, a point stressed by Lumb (1960), and this method was abandoned.

Received for publication 4 September 1975
Alternatively, sections from surgical partial and total colectomy and proctocolectomy specimens at King's College Hospital and Kingston Hospital were studied, covering the years 1965-74 and 1958-74 respectively, and examined for the presence of $\mathrm{L}-\mathrm{G}$ complexes. The site of excision, age, sex, and pathological diagnosis of each specimen were noted from surgical reports, day books, and, when necessary, from case notes.

\section{Results}

Altogether sections from 1924 specimens were examined. One or more L-G complexes were found in sections from 231 specimens. Of these, 33 were present in specimens of caecum and ascending colon, nine in specimens of transverse colon, and 172 in specimens of descending colon and rectum. L-G complexes were present in 17 total colectomy specimens but the sites from which the blocks were taken were not ascertainable. The distribution of L-G complexes and the number of each type of specimen examined are shown in the table. Of the 17 total colectomy specimens showing L-G complexes, 15 were from cases of ulcerative colitis, one of Crohn's disease, and one of diverticular disease. The disease processes in which L-G complexes occurred are as follows: primary carcinoma, carcinoma with diverticular disease, diverticular disease, ulcerative colitis, Crohn's disease, non-specific inflammation, polyps, intussusception, volvulus, Hirschsprung's 


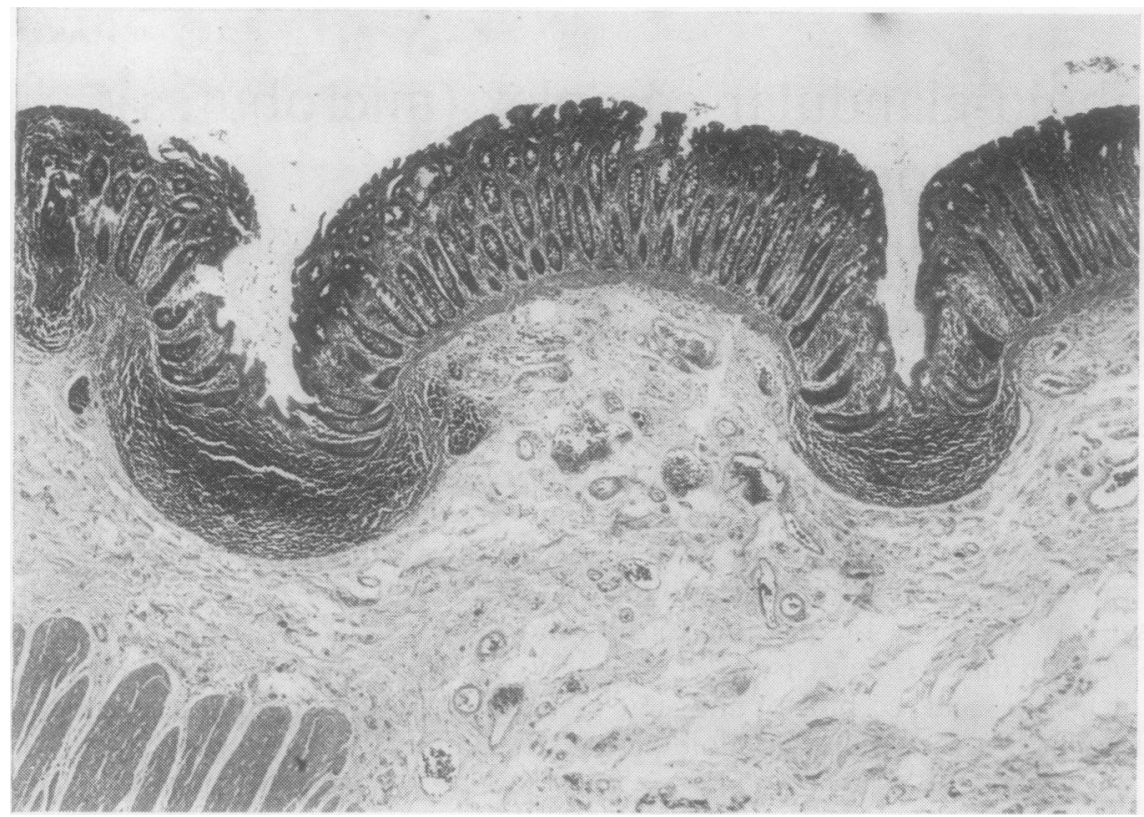

Fig 1 Colon showing two $L-G$ complexes ( $H$ and $E \times 30$ ).

\begin{tabular}{lcccc}
\hline & $\begin{array}{l}\text { Caecum } \\
\text { and } \\
\text { Ascending } \\
\text { Colon }\end{array}$ & $\begin{array}{l}\text { Transverse } \\
\text { Colon }\end{array}$ & $\begin{array}{l}\text { Descending Total } \\
\text { Colon and } \\
\text { Rectum }\end{array}$ & \\
& Colectomy \\
\hline No. of specimens & 383 & 73 & 1397 & 71 \\
No. of L-G complexes & 33 & 9 & 172 & 17 \\
Percentage incidence & $8 \cdot 6$ & $12 \cdot 3$ & $12 \cdot 3$ & 23.9 \\
\hline
\end{tabular}

Table Number of specimens examined and those showing L-G complexes

disease, and vascular lesions. They were found also in two specimens which showed no gross or microscopic abnormality, one from a 1-year-old girl with imperforate anus, from whom a short length of descending colon was removed, and the other from a 34-year-old man from whom the caecum and part of the ascending colon were excised.

The youngest specimen in which L-G complexes were present was from the female child mentioned previously; the oldest was from a woman of 86 with carcinoma of the rectum. L-G complexes were present in specimens from all age groups between 1 year and 86 years, though sections from 12 specimens in the age group 91-100 did not reveal any.

SITES OF L-G COMPLEXES

Serial sections through several separate surgical blocks of colon, both parallel and perpendicular to the mucosa, showed that the gaps in the muscularis mucosae are true defects filled with lymphoid tissue, and lymphocytes could be seen between the fibres of the adjacent muscularis mucosae (fig 2). Lymphocytes were also seen inside the epithelial cytoplasm of some L-G complexes.

Measurement of the ratios of the maximum transverse diameters of the lymphoid nodules to the widths of the gaps in the muscularis mucosae showed that increased size of the lymphoid nodules was paralleled by an increase in width of the gap. No correlation was demonstrated between the extent of protrusion of the mucosa through the gaps with the diameters of the lymphoid nodules and the widths of the gaps in the muscularis mucosae.

\section{Discussion}

The results show that L-G complexes occur throughout the whole length of the large bowel, that they occur in normal and disease states, and that they are present in all age groups. Dyson makes the point that these structures are herniations of mucosal epithelium through gaps in the muscularis mucosae and that the initiating factor in their formation is likely to be sustained contraction of the muscularis mucosae, thus suggesting that they are acquired.

The increase in number of $\mathbf{L}-\mathrm{G}$ complexes in sections of ulcerative colitis and Crohn's disease may be more apparent than real, as in these conditions the protrusion of mucosa through gaps in the 


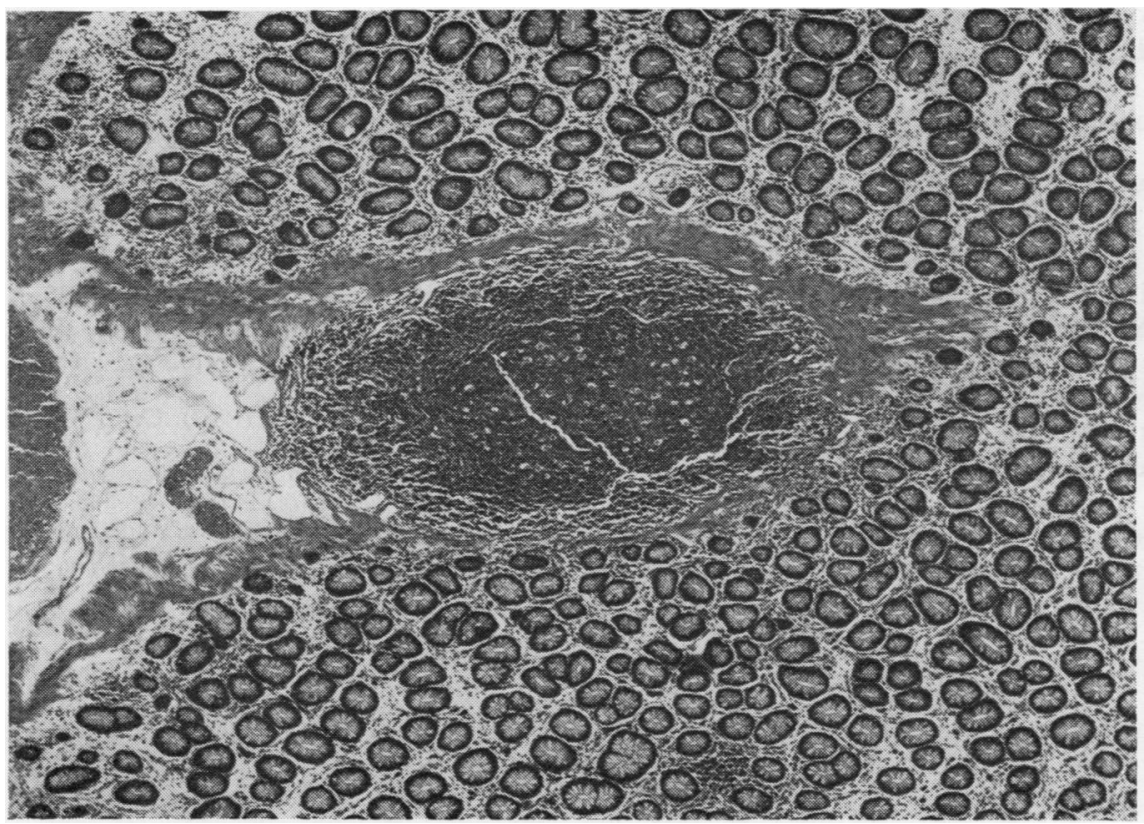

Fig 2 Section taken in the same plane as the surface of the mucosa showing a lymphoid nodule inside a gap in the muscularis mucosae ( $H$ and $E \times 30$ ).

muscularis mucosae may not represent true L-G complexes, as they are often unassociated with lymphoid nodules and are possibly foci of re-epithelialization following destruction of the muscularis mucosae in these inflammatory states. Histological examination of L-G complexes often showed degenerative changes in some of the constituent glands. In no case, however, was cellular atypicality sufficient to warrant a diagnosis of carcinoma. But, in one case of carcinoma, malignant epithelium could be seen streaming through a nearby gap in the muscularis mucosae into the underlying lymphoid nodule, which may partly explain the occurrence of early metastases from some small carcinomas of the colon.

Cases of colitis cystica profunda, a rare disease of the colon associated with the presence of epitheliallined mucus-filled cysts in the submucosa, were described by Goodall and Sinclair (1957), who thought that the cysts developed from epithelium which had extended into the submucosa during the healing of small deep follicular ulcers following necrosis of the submucosal lymphoid nodules. Wayte and Helwig (1967) considered that the cysts resulted from extension of surface mucosa along granulation tissue tracts created through the muscularis mucosae. Epstein et al (1966) thought that they may develop from herniation of mucosa through a muscularis mucosae congenitally weak or damaged by inflammation or perhaps by re-epithelialization of deep undermining ulcers. The latter authors noted that the cysts were never observed in association with lymphoid nodules. It would seem therefore that L-G complexes, which occur commonly, which are present in all age groups, in all parts of the large bowel, are entities distinct from the cysts of colitis cystica profunda.

What then is the nature of these L-G complexes? It seems likely that they are normal structures of the bowel and are the immunological watchdogs which can be easily reached by antigenic material present in the bowel lumen and which can gain easy access to the lymphoid tissue through the gaps in the muscularis mucosae and across the single layer of epithelium that separates it from the underlying lymphoid nodule. Structures morphologically similar to L-G complexes are present in the small bowel. An analogy may be drawn between L-G complexes and the crypts of the palatine tonsils which are lined by stratified squamous epithelium and dip into the lymphoid tissue, rendering the tonsils more effective immunologically. The bursa of Fabricius, a round or pear-shaped lymphoid diverticulum in the hindgut of birds, lined by pseudostratified epithelium, bears some morphological resemblance to the L-G complex when viewed in this context. It is suspected that mammals have an equivalent tissue, perhaps more widely distributed and forming the lymphoid tissue 
of the appendix, the colon, and Peyer's patches of the small intestine (Walter and Israel, 1974). Microbursae rather than L-G complexes may be a more apt name for these structures.

I wish to thank Dr M. E. A. Powell, Kingston Hospital, for criticism and advice; Professor E. A. Wright, King's College Hospital and Dr J. H. Earle, Queen Mary's Hospital, Roehampton, for access to surgical sections; Mr J. Spicer, Kingston Hospital, for technical assistance; and Miss B. R. Hume for typing this paper. The work was supported by a grant from the South West Thames Regional Health Authority for the purchase of photomicrographic equipment.

This paper constitutes part of the work for an MD thesis to be submitted to the National University of Ireland.

\section{References}

Clark, R. M. (1969). Microdiverticula: a possible cause of granulomatous ileocolitis. Canad. med. Ass. J., 100, 1025-1031.

Clark, R. M. (1970). Microdiverticula and submucosa epithelial elements in ulcerative and granulomatous diseases of the ileum and colon. Canad. med. Ass. J., 103 24-28.

Dyson, J. L. (1975). Herniation of mucosal epithelium inton the submucosa in chronic ulcerative colitis. J. clin. Path., 28, 189-194.

Epstein, S. E., Ascari, W. Q., Ablow, R. C., Seaman, W. B. and Lattes, R. (1966). Colitis cystica profunda. Amer. $J_{\vec{\omega}}$ clin. Path., 45, 186-201.

Goodall, H. B., and Sinclair, I. S. R. (1957). Colitis cystic profunda. J. Path. Bact., 73, 33-42.

Lumb, G. (1960). Normal human rectal mucosa and itફ mechanism of repair. Amer. J. dig. Dis., 5, 836-840.

Walter, J. B. and Israel, M. S. (1974). General Pathology, 4thu edition, p. 149. Churchill Livingstone, Edinburgh.

Wayte, D. M., and Helwig, E. B. (1967). Colitis cysticap profunda. Amer. J. clin. Path., 48, 159-169. 